increased risk of liver events in the 90 days immediately prior to and post first Orlistat prescription, but no difference in risk between the pre and initial exposure periods. This suggests that Orlistat may be initiated during a period of time when adverse liver events are more likely due to poor underlying health, but does not suggest the risk increases with initiation of Orlistat.

\section{OP94 EVIDENCE FOR THE EFFECTIVENESS OFOPIATE SUBSTITUTION TREATMENT IN RELATION TO HIV TRANSMISSION IN PEOPLE WHO INJECT DRUGS: A SYSTEMATIC REVIEW AND META-ANALYSIS}

doi:10.1136/jech-2012-201753.094

${ }^{1} \mathrm{GJ}$ MacArthur, ${ }^{2} \mathrm{~S}$ Minozzi, ${ }^{1,3} \mathrm{~N}$ Martin, 1,3P Vickerman, ${ }^{4} \mathrm{~J}$ Bruneau, ${ }^{2} \mathrm{M}$ Davoli, ${ }^{1} \mathrm{M}$ Hickman. 'School of Social and Community Medicine, University of Bristol, Bristol, UK; ${ }^{2}$ Department of Epidemiology, Lazio Regional Health Service, Rome, Italy, ${ }^{3}$ Centre for Research on Drugs and Health Behaviour, LSHTM, London, UK; ${ }^{4}$ Research Center, Centre Hospitalier de I'Université de Montréal, Montreal, Canada

Background Injecting drug use is a major risk factor for the acquisition and transmission of HIV among people who inject drugs (PWID), and between PWID and the wider community. Worldwide there are an estimated 15.9 million PWID of whom 3 million may be HIV-positive. Methadone and buprenorphine (opiate substitution treatments, OST) reduce heroin use, injecting risk behaviour, and drug related mortality and are included in the World Health Organization list of essential medicines. A small number of individual cohort studies and a Cochrane narrative systematic review suggest that OST may reduce HIV incidence, but no pooled quantitative synthesis has been carried out. We have undertaken a systematic review and meta-analysis of published and unpublished studies to quantify the effect of OST on HIV transmission.

Methods Medline, EMBASE and PsychINFO were searched to October 2011 to identify studies that examined the effectiveness of OST in relation to HIV transmission. Authors of prospective studies that assessed HIV incidence in PWID were contacted to obtain unpublished data.

Results Fifteen studies conducted in seven countries were relevant for inclusion. Data from ten of the studies were pooled, two of which were unpublished. Analysis included over 22,000 personyears of follow-up and 738 incident HIV infections. Preliminary random effects meta-analysis demonstrates that OST reduces risk of HIV transmission among PWID by $49 \%$ (RR 0.51 , 95\% CI $0.37-$ $0.71 ; \mathrm{p}<0.001)$ although there was significant heterogeneity between studies (I $59.5 \%, \chi^{2} 22.2, \mathrm{p}=0.008$ ). Study-level covariates including publication year, gender, median age, and ethnicity of participants did not significantly influence the impact of OST in meta-regression analyses. However, sub-group analysis demonstrated that whilst continuous OST significantly reduced risk of HIV infection, the effectiveness of interrupted or detoxification treatment was less clear (RR 1.26, 95\% CI 0.77-2.07; $\mathrm{p}=0.360$ ).

Conclusion These preliminary data provide further evidence that OST can reduce the risk of HIV infection among PWID and for the first time quantify the effect. Ensuring sufficient coverage of OST as part of a package of harm reduction interventions is critical to reduce the burden of HIV among PWID and to prevent onward transmission between PWID and to the wider community.

\section{OP95 RISK FACTORS FOR FIRST VENOUS THROMBOEMBOLISM IN AND AROUND PREGNANCY: A POPULATION BASED COHORT STUDY FROM THE UNITED KINGDOM}

\section{doi:10.1136/jech-2012-201753.095}

${ }^{1} \mathrm{~A}$ Abdul Sultan, 'LJ Tata, ${ }^{1} \mathrm{~J}$ West, 'L Fiaschi, ${ }^{1} \mathrm{KM}$ Fleming, ${ }^{2} \mathrm{C}$ Nelson-Piercy. ${ }^{1} \mathrm{Di}-$ vision of Epidemiology and Public Health, University of Nottingham, Nottingham, UK; 'Women's Health Academic Center, Guy's \& St Thomas' Foundation Trust, St Thomas' Hospital, London, UK
Background Venous thromboembolism (VTE) remains one of the leading causes of maternal mortality in high income countries. A lack of robust data on women's risk factors for antepartum and postpartum VTE limit potential prevention. There is a need for estimates of absolute risks at population level according to recognised risk factors.

Methods Using a large primary care database, we analysed 376,154 pregnancies ending in live births or stillbirths from women 15-44 years of age between 1995 and 2009. We assessed the impact of risk factors on the absolute and relative incidence of VTE for antepartum and postpartum periods using Poisson regression.

Results Postpartum, the strongest risk factor was stillbirth (Absolute VTE Rate $=2,444 / 100,000$ person-years) followed by varicose veins, BMI $>30 \mathrm{~kg} / \mathrm{m} 2$, obstetric haemorrhage, preterm delivery, medical co-morbidities (either SLE, IBD, nephrotic syndrome or cancer) and caesarean section ( $A R=637 / 100,000$ person-years or higher). $\mathrm{BMI}>30 \mathrm{~kg} / \mathrm{m} 2$ conferred a substantial increase in postpartum risk ( $A R=926 / 100,000$ person-years) but only a modest increase antepartum ( $A R=109 / 100,000$ person-years). Women age $>35$ years, current smokers, and those with acute systemic infections had small relative increases in antepartum and postpartum VTE to those without such risk factors.

Conclusion Antepartum VTE varies modestly by recognised risk factors, yet women with stillbirths, preterm births, obstetric haemorrhage, caesarean section delivery, co-morbidities or BMI $>30 \mathrm{~kg} /$ $\mathrm{m} 2$ are most likely to benefit from thromboprophylaxis postnatally. For example, we estimate that up to 17 to 159 annual VTEs could be avoided annually if all women with stillbirth, preterm birth or caesarean section in the UK received appropriate thromboprophylaxis.

\section{OP96 SURVEY OF USE AND APPLICATION OF TEST ACCURACY MEASURES FOR DECISION MAKING IN PRIMARY CARE}

doi:10.1136/jech-2012-201753.096

${ }^{1}$ CF Davenport, ${ }^{2} \mathrm{CJ}$ Hyde. ${ }^{1}$ Public Health, Epidemiology and Biostatistics, University of Birmingham, Birmingham, UK; ${ }^{2}$ Penninsula Technology Assessment Group, University of Exeter, Exeter, UK

Background Increase in test use over recent decades has occurred despite disappointing results from test accuracy evaluations. Difficulties with understanding and application of test accuracy information are purported to be important contributors to this observed evidence 'gap'. Empirical research to date is based on the premise that formal probability revision is a necessary pre-requisite for informed diagnostic decison making and is characterised by self selected samples with recent experience or expertise in test evaluation. The survey aims were to describe how clinicians apply existing test accuracy metrics for diagnostic decision making.

Methods An incentivised, electronic survey was used. Informed application of test accuracy information was evaluated by asking respondents to indicate their management decision following presentation of nine different representations of the same test accuracy information to a common hypothetical scenario. Quantitative and qualitative synthesis was employed based on closed and open responses to management decisions.

Results A total of 204 General Practitioners (response rate 95\%) did not appear to be self-selected on the basis of academic position, involvement in policy or experience in test evaluation. Sensitivity and specificity, the annotated $2 \times 2$ diagnostic table and predictive values were reported to be familiar metrics by the most respondents. Likelihood ratios the DOR and AUC were familiar to less than $1 / 3$ of respondents. Application of test accuracy metrics resulted in marked variation in responses to both positive and negative test results although greater inconsistency and management uncertainty was observed following presentation of a negative test result. Formal probability revision was not a feature of the diagnostic decision making process. Test errors 
(false negatives and false positives) were prominent as part of the translational pathway from quantitative summary estimates of test accuracy to management decisions. Summary measures that separate the two dimensions of test accuracy in the absence of prevalence information (for example sensitivity and specificity) appeared to result in a misplaced emphasis on one or other of false positive or false negative test errors. Presenting test accuracy data using the $2 \times 2$ diagnostic table or a pictograph attenuated this effect.

Conclusion Choice of test accuracy metric appears to have a profound effect on diagnostic decision making. Understanding, contextual factors and motivational biases are likely to be contributing factors to the observed variability. It is unclear to what extent any advantage of test accuracy metric for informed decision making is based on familiarity as opposed to their intuitive nature. Simultaneous illustration of both dimensions of test accuracy in order to facilitate informed diagnostic decision making requires further exploration.

\section{Plenary Session}

\section{PL01 A SIMPLE MORBIDITY SCORE FOR UK PRIMARY CARE: A NEW TOOL FOR RESEARCH AND HEALTHCARE OUTCOME MONITORING}

doi:10.1136/jech-2012-201753.097

'IM Carey, 'SM Shah, 'T Harris, 'S DeWilde, 'DG Cook. 'Division of Population Health Sciences and Education, St George's University of London, London, UK

Background Adjustment for morbidity level is important in ensuring fair comparison of outcomes between patient groups and healthcare providers. The Quality and Outcomes Framework (QOF) in UK primary care, which records numerous diseases systematically, offers potential for developing a standardised morbidity score that can be easily applied in research and service settings.

Methods Using The Health Improvement Network (THIN), a large primary care database of 375 UK general practices in 2008-9, half the practices were randomly selected as a training set to derive a morbidity score based on chronic conditions recorded in QOF, and the other practices formed a validation set to assess predictive performance. A total of 653,780 patients aged 60 and over registered in 2008 were included, and mortality at one year was assessed.

Results Nine QOF conditions were identified as robust co-predictors (Hazard Ratio $\geq 1.2$ ) of mortality independent of age and sex, and were assigned integer score weights based on the strength of their association with mortality. Cancer $(\mathrm{HR}=3.4)$ and Dementia $(\mathrm{HR}=2.8)$ were the strongest predictors. In a Cox model with age and sex included, the addition of the QOF score improved model discrimination in predicting mortality (c-statistic $=0.82 \mathrm{vs}$. 0.78 ), performing similarly to the Charlson index, an established morbidity index. In a multilevel logistic model, an individual's QOF score explained more of the variation in mortality between practices than the Charlson index (46\% compared to 32\%). At practice level, the mean QOF score per patient was strongly correlated with practice standardised mortality ratios $(r=0.64)$ and explained more variation in practice death rates than the Charlson index.

Conclusion A simple score derived from routine QOF recording provides a morbidity index which is highly predictive of one year mortality in older UK Primary Care patients, is simpler to implement than existing morbidity scores, and explains practice level variations in mortality. This new score has potential utility in research and healthcare outcome monitoring and could be easily implemented nationally through existing mechanisms for anonymised collection of QOF data from practices.

\section{PLO2 BREASTFEEDING AND SOCIAL MOBILITY: NEUROLOGICAL DEVELOPMENT OR STRESS MECHANISMS?}

doi:10.1136/jech-2012-201753.098

${ }^{1} \mathrm{~A}$ Sacker, ${ }^{1} \mathrm{Y}$ Kelly, ${ }^{2} \mathrm{M}$ Bartley, ${ }^{1} \mathrm{M}$ lacovou. ${ }^{1}$ Institute for Social and Economic Research, University of Essex, Colchester, UK; ${ }^{2}$ Research Department of Epidemiology and Public Health, UCL, London, UK

Background Breastfeeding has been associated with higher cognitive scores, better test results and fewer socio-emotional problems in childhood. These outcomes in turn predict social mobility. This study examines the effect of breastfeeding on inter-generational social mobility and the role of two biologically plausible mechanisms: via improved neurological development due to the longchain polyunsaturated fatty acids in breast milk and via hypothalamic-pituitary-adrenal (HPA) axis functioning due to growth hormones in breast milk.

Methods We use data from two birth cohorts (the 1958 National Child Development Study and the 1970 British Birth Cohort) to examine breastfeeding's relationship with social mobility. Social class at 10/11 years was based on father's class. Both class of origin and own social class in adulthood (age 33/34) were measured by the Registrar General's social class (RGSC). Neurological development was assessed using cognitive tests and assessments of fine motor function. HPA functioning was assessed using socio-emotional behaviour scales and a physical symptoms of stress score.

Results Rates of breastfeeding were higher in the 1958 cohort than the 1970 cohort (43\% vs. $21 \%$ breastfed $1+$ months). Breastfeeding was more socially patterned by 1970 , with advantaged mothers being more likely to breastfeed. A propensity score approach matched breastfed and non-breastfed children on a large number of characteristics before estimating the effect of breastfeeding on social mobility. We modelled the odds of upward and downward social mobility conditional on being breastfed for 1 month or more and social class of origin. Results show that breastfeeding increased the odds of upward mobility (1958 cohort: OR 1.25 95\% CI 1.13, 1.37; 1970 cohort: OR 1.14 95\% CI 1.00, 1.31), and reduced the odds of downward mobility (1958 cohort: OR 0.81 $95 \%$ CI $0.74,0.90 ; 1970$ cohort OR 0.79 95\% CI 0.69, 0.91). Controlling for the measures of neurological development and stress functioning attenuated the effect of breastfeeding to marginal or non significance. Gender differences in these findings will be discussed

Conclusion Breastfeeding promotes upward social mobility and protects against downward mobility. The effects appear to operate through enhanced neurological development and more effective stress processes.

\section{PL03 SOCIO-ECONOMIC INEQUALITIES IN LUNG CANCER TREATMENT: A SYSTEMATIC REVIEW AND META- ANALYSIS}

doi:10.1136/jech-2012-201753.099

${ }^{1} \mathrm{LF}$ Forrest, $1 \mathrm{JM}$ Adams, ${ }^{2} \mathrm{H}$ Wareham, ${ }^{2} \mathrm{G}$ Rubin, ${ }^{1} \mathrm{M}$ White. ${ }^{1}$ Institute of Health and Society, Newcastle University, Newcastle upon Tyne, UK; ${ }^{2}$ Wolfson Research Institute, Durham University (Queen's campus), Stockton on Tees, UK

Background Intervention-generated inequalities in health result from the way that health interventions are organised and delivered. There is some evidence that socio-economic inequalities in care may occur for some common cancers and treatment inequalities may contribute to socio-economic differences in survival. Although the incidence and outcome of lung cancer varies with socio-economic status (SES), it is not known whether socio-economic inequalities in treatment occur. We conducted a systematic review and metaanalysis of existing research on socio-economic inequalities in receipt of treatment for lung cancer. 